\title{
Spectropolarimetric measurements of the mean longitudinal magnetic field of chemically peculiar stars ${ }^{\star}$
}

\section{Phase relating the magnetic and luminosity variabilities}

\author{
F. Leone ${ }^{1}$ and G. Catanzaro ${ }^{2}$ \\ 1 Osservatorio Astrofisico di Catania, Città Universitaria, 95125 Catania, Italy \\ 2 Center for Astrophysical Sciences, The Johns Hopkins University, Dept. of Physics and Astronomy, Bloomberg \\ Center, 3400 N. Charles str., Baltimore, MD 21218, USA
}

Received 21 June 2000 / Accepted 19 September 2000

\begin{abstract}
For a sample of chemically peculiar stars, we report time-resolved measurements of the effective magnetic field which were obtained with the spectropolarimetry operating at the Catania Astrophysical Observatory. These observations are combined with data from the literature for better pointing out that periodic magnetic variability which characterises this class of stars. Periods given in the literature have been checked and, if possible, redetermined, not only by means of the magnetic measurements but referring also to the Hipparcos photometry. The variability of the effective magnetic field of the already known magnetic star 25 Sex is pointed out for the first time. As to the suspected magnetic chemically peculiar star EP UMa, our measurements confirm that this is really a magnetic star and we indicate a possible variability period. The accuracy of the variability period for CS Vir and FF Vir is improved. The suggestion that light variability is due to the re-distribution of ultraviolet flux towards the visible wavelengths in metal rich regions, which are not homogeneously distributed on the stellar surface, appears not always and straightly valid. Local line-blocking is certainly important in the case of CS Vir and a direct influence of the magnetic field on the infrared photometric variability cannot be ruled out for 25 Sex.
\end{abstract}

Key words. stars: chemically peculiar - stars: magnetic fields

\section{Introduction}

Magnetic Chemically Peculiar (CP) stars show periodic spectral, light and magnetic variability. From Babcock (1949) and Stibbs (1950), these stars are commonly understood within the Oblique Rotator Model, consisting of: i) a large scale organised magnetic field whose symmetry axis, if any, is tilted with respect to the rotational axis; and ii) a non uniform distribution of chemical elements on the stellar surface. Variability is due to the stellar rotation.

Since it is not yet possible to resolve a stellar disk, several observational quantities and algorithms are necessary to determine the morphology of magnetic fields and the surface-distribution of elements of CP stars:

- Taking advantage of the properties of Zeeman components, we have the possibility to perform polarimetric measurements that give the average over the stellar disk of the longitudinal components of the field, the

Send offprint requests to: F. Leone,

e-mail: fleone@sunct.ct.astro.it

* Based on observations collected at the Catania Astrophysical Observatory, Italy. so called effective magnetic field $H_{\text {eff }}$. From the circularly polarised light spectroscopy by Babcock (1947) and photometry by Borra \& Landstreet (1973), the relatively easy measurement of $H_{\text {eff }}$ is, up to now, the most common method to detect stellar magnetic fields. Introduced by Mathys (1993), the moment technique relates the line asymmetries of the Stokes $V$ parameters to the distribution of magnetic fields on the stellar surface. Very recently, based on high efficiency of modern astronomical instruments, quantities related to the average over the stellar disk of the transverse components of the field have been measured by means of linearly polarised light photometry (Landolfi et al. 1993) and spectroscopy (Borra \& Vaughan 1976; Wade et al. 2000);

- High resolution spectroscopy is a further important diagnostic tool to recover the magnetic field geometry. From Babcock (1960), it is possible to measure the average on the visible disk of the magnetic field modulus $\left(H_{\bmod }\right)$. Moreover, high resolution spectroscopy gives information on the element distribution over the stellar surface; 
Table 1. Observed magnetic chemically peculiar stars and their measured effective magnetic fields with the associated errors. Spectral types and peculiarity classes are from Renson et al. (1991), $V$ magnitudes from SIMBAD database

\begin{tabular}{|c|c|c|c|c|c|c|c|c|c|c|c|}
\hline $\begin{array}{r}\text { JD } \\
2,451,000+ \\
\end{array}$ & $\begin{array}{r}H_{\text {eff }} \\
\mathrm{G} \\
\end{array}$ & $\begin{array}{l}\sigma \\
\mathrm{G} \\
\end{array}$ & & & & & & & & & \\
\hline $53 \mathrm{Cam}$ & & & $25 \mathrm{Sex}$ & & & EP UMa & & & & & \\
\hline $\mathrm{A} 3 \mathrm{SrEuCr}$ & $V=6.0$ & & B9SiCrSr & $V=5.92$ & & B8Sr $\quad V$ & $=6.08$ & & & & \\
\hline 262.369 & 3130 & 450 & 213.542 & 580 & 180 & 218.581 & -570 & 300 & & & \\
\hline 264.376 & -3335 & 470 & 214.542 & -955 & 500 & 237.524 & -40 & 560 & & & \\
\hline \multirow[t]{4}{*}{265.325} & -4570 & 560 & 218.534 & 150 & 270 & 238.517 & -500 & 270 & & & \\
\hline & & & 237.478 & -1140 & 460 & 262.464 & 930 & 600 & & & \\
\hline & & & 265.373 & 1110 & 100 & 264.477 & 790 & 100 & & & \\
\hline & & & & & & 265.466 & -150 & 500 & & & \\
\hline 78 Vir & & & CS Vir & & & FF Vir & & & $\beta \mathrm{CrB}$ & & \\
\hline $\mathrm{A} 1 \mathrm{EuCr}$ & $V=4.91$ & & $\mathrm{~A} 9 \mathrm{SrEuCr}$ & $V=5.8$ & & $\mathrm{~A} 9 \mathrm{CrSr}$ & $V=4.12$ & & $\mathrm{~A} 8 \mathrm{SrEu}$ & $V=3.68$ & \\
\hline 262.514 & -890 & 330 & 262.588 & 1940 & 330 & 218.674 & 1680 & 620 & 264.678 & 555 & 180 \\
\hline 264.551 & -20 & 230 & 265.612 & -710 & 330 & 237.621 & 2030 & 800 & 265.644 & 785 & 310 \\
\hline 265.541 & -480 & 210 & 304.507 & -2190 & 400 & 238.622 & 2040 & 470 & 299.528 & 110 & 290 \\
\hline 303.431 & -750 & 320 & 305.515 & -1560 & 250 & 264.632 & 370 & 290 & 304.606 & 580 & 300 \\
\hline 305.443 & -370 & 340 & 328.454 & 2230 & 540 & 303.517 & -2100 & 710 & 305.548 & 670 & 280 \\
\hline 323.397 & -590 & 400 & 357.372 & 1220 & 500 & 325.426 & -610 & 450 & 323.463 & 980 & 300 \\
\hline 324.339 & 20 & 280 & 358.338 & -760 & 110 & & & & 326.511 & 640 & 250 \\
\hline 325.369 & -560 & 420 & 361.349 & -1600 & 640 & & & & 328.509 & -120 & 200 \\
\hline 326.357 & -1250 & 320 & 363.348 & 665 & 150 & & & & 329.479 & -200 & 320 \\
\hline 328.359 & -185 & 210 & 364.346 & 1840 & 600 & & & & 355.589 & 330 & 280 \\
\hline \multirow[t]{3}{*}{329.324} & -760 & 400 & & & & & & & 359.518 & 760 & 240 \\
\hline & & & & & & & & & 363.512 & 800 & 240 \\
\hline & & & & & & & & & 367.555 & -460 & 190 \\
\hline
\end{tabular}

- Introduced by Deutsch (1947) to infer the magnetic field geometry from observed quantities, inversion procedures had a great progress thanks to Landolfi et al. (1998). Who represented the magnetic field as the superposition of a dipole plus a quadrupole and presented an algorithm to invert the observational data. This procedure has been successfully applied by Bagnulo et al. (1999);

- The determination of rotational periods is clearly a primary step to invert the magnetic observable quantities and combine observations that were not simultaneously obtained. In the Oblique Rotator Model, stellar rotational periods can be determined easily and with very high accuracy from photometric variations;

- Assuming that the star is a rigid rotator, the knowledge of the stellar radius, when measured from absolute magnitudes (North 1998), strongly constrains the projected rotational velocity in inversion procedures.

Within the previous scenario, for a sample of CP stars we present spectropolarimetric measurements that were obtained with the aim to establish the periodic variability of $H_{\text {eff }}$.

\section{Observational data and analysis}

For the chemically peculiar stars, listed in Table 1, we have carried out time-resolved spectropolarimetric observations at the Catania Astrophysical Observatory in 1999 and measured the effective magnetic field. The instrument, data reduction and analysis are described by Leone et al. (2000).

Based on spectra with signal-to-noise ratio larger than 100, results are listed in Table 1 too.

For each star, our results are combined with data from the literature for better pointing out the $H_{\text {eff }}$ variability. According to the oblique rotator model and whenever possible, we have used photometric data to improve the accuracy of period $P$. Particularly, Hipparcos magnitudes ( $V_{\text {Hip }}$, European Space Agency 1997) have been analysed. To perform period searching and establish phase relations, a least-square fitting of magnetic and photometric data have been performed with the function:

$$
\begin{aligned}
f= & A_{0}+A_{1} \sin 2 \pi\left(\frac{\left(t-t_{0}\right)}{P}+\phi_{1}\right) \\
& +A_{2} \sin 2 \pi\left(\frac{2\left(t-t_{0}\right)}{P}+\phi_{2}\right)
\end{aligned}
$$

where $t$ is the time of observations and $t_{0}$ is a reference instant.

To over-plot differential photometry light curves given in different authors, we have determined the $A_{0}$ values by fitting each data set with the previous relation. In presence of absolute magnitudes, we have shifted the differential photometric data. 


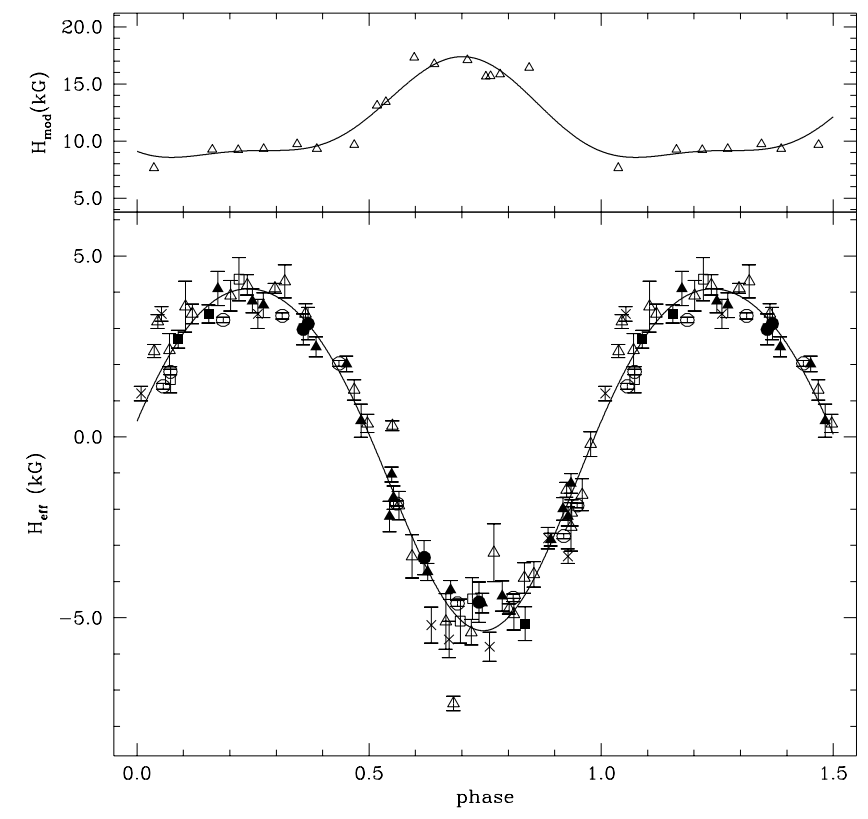

Fig. 1. Modulus and effective magnetic field of 53 Cam as a function of the rotational phase. As to $H_{\text {eff }}$, photopolarimetric measurements are by Landstreet et al. (1975), Borra \& Landstreet (1977), Borra et al. (1984) (open triangles) and Hill et al. (1998) (filled triangles). Spectropolarimetric measurements based on photographic plates are by Preston \& Stepień (1968) (crosses), on CCD are by Elkin (1996) (filled squares), Hildebrandt et al. (1997) (open squares), Wade et al. (2000) (empty circles) and by us (filled circles). The magnetic field modulus was measured by Mathys et al. (1997). Errorbars are equal to two times the measurement errors. Solid lines represent a least-square fit with Eq. (1)

\section{Individual stars}

\subsection{Cam $=H D 65339=H R 3109=H I P 39261$}

$53 \mathrm{Cam}$ is classified as an $\mathrm{A} 3 \mathrm{SrEuCr}$ star in the General Catalogue of Ap and Am stars by Renson et al. (1991).

Jarzebowski (1960a, 1960b) firstly established the light variability of $53 \mathrm{Cam}$ at $\lambda_{\mathrm{eff}}=4200$ and $5350 \AA$. Adopting the 8.0248 day period, this author found that the previous two light curves are not in phase and that the effective magnetic field, measured by Babcock, presents a minimum close in phase to the $\lambda_{\text {eff }}=4200 \AA$ minimum. Preston \& Stepień (1968) measured the effective magnetic field and obtained photometric observations in the $U B V$ system. They determined a variability period equal to 8.0278 days and found that the $U$ and $B$ variations are in phase with the magnetic field.

Further $U B V$ observations of 53 Cam have been obtained by Stepień (1978) and Ten Colour Photometry were carried out by Musielok et al. (1980).

On the basis of effective magnetic field measurements, Hill et al. (1998) determined the ephemeris:

$\mathrm{JD}($ crossover $)=2448498.186+8.02681 E$.

Figure 1 reports our $H_{\text {eff }}$ measurements with the photopolarimetric measurements by Landstreet et al. (1975),
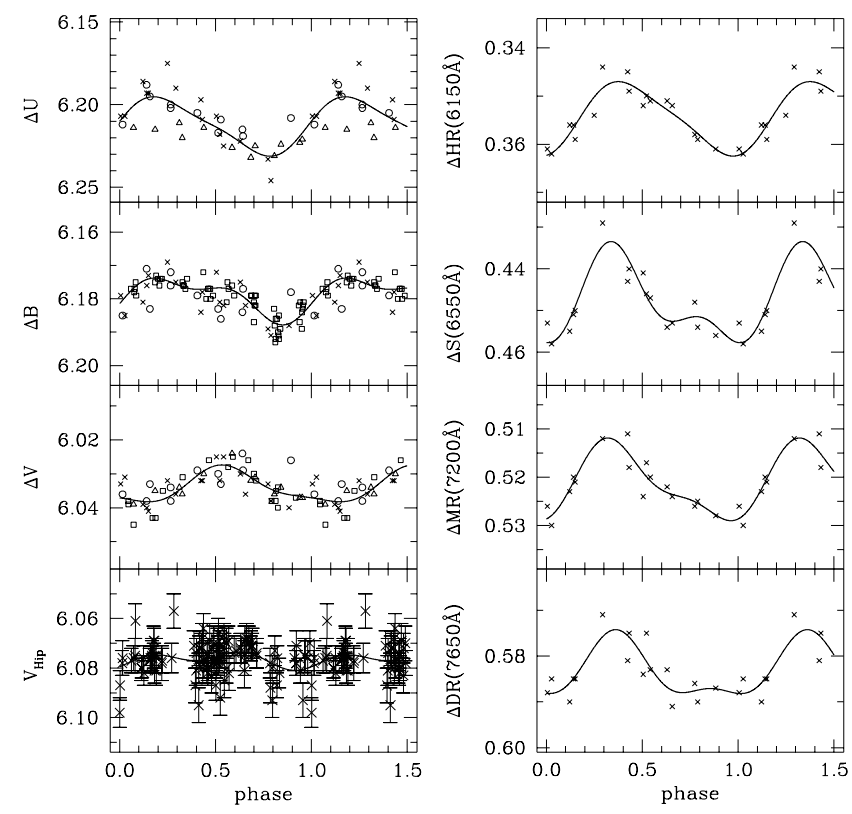

Fig. 2. Light curves of 53 Cam. Squares represent Jarzebowski (1960a, 1960b) differential photometry appropriately shifted. $U B V$ photometry is by Preston \& Stepień (1968) (circles) and Stepień (1978) (triangles). Crosses represent the Ten Colour Photometry by Musielok et al. (1980). Light curves change so significantly with the wavelength that Hipparcos light curve is almost constant because its very large pass-band filter

Borra \& Landstreet (1977), Borra et al. (1984) and Hill et al. (1998); the spectropolarimetric measurements based on photographic plates by Preston \& Stepień (1968) and on CCD by Elkin (1996), Hildebrandt et al. (1997) and Wade et al. (2000). Our measurements confirm the validity of Hill et al. period. The $H_{\text {eff }}$ variability is almost sinusoidal with the $A_{2}$ term of Eq. (1) negligible with respect to $A_{1}$.

In the same figure are also plotted Mathys et al. (1997) measurements of the magnetic field modulus, whose variability shows an important second harmonic.

We have also phased all the previously quoted photometric data with Hill et al. (1998) period (Fig. 2). We note that the photometric curves of 53 Cam changes significantly with the wavelengths. The minimum of the $U$ light curve shows the phase of $H_{\text {eff }}$ minimum and the $\Delta H R$ (6150 $\AA$ ) light curve minimum coincides with the phase of the $H_{\text {eff }}$ null. Longward of this wavelength, the $A_{2}$ term of Eq. (1) is comparable to $A_{1}$. Unfortunately, time-resolved photometry in the infrared is not available for 53 Cam to establish the further variations of light curves with the wavelength.

Changing the light-curve minimum with the wavelength within the very large $(F W H M=2200 \AA)$ passband of the $H_{\mathrm{p}}$ filter (whose maximum is at $4500 \AA$ ), the Hipparcos light curve of $53 \mathrm{Cam}$ is almost constant (Fig. 2). 

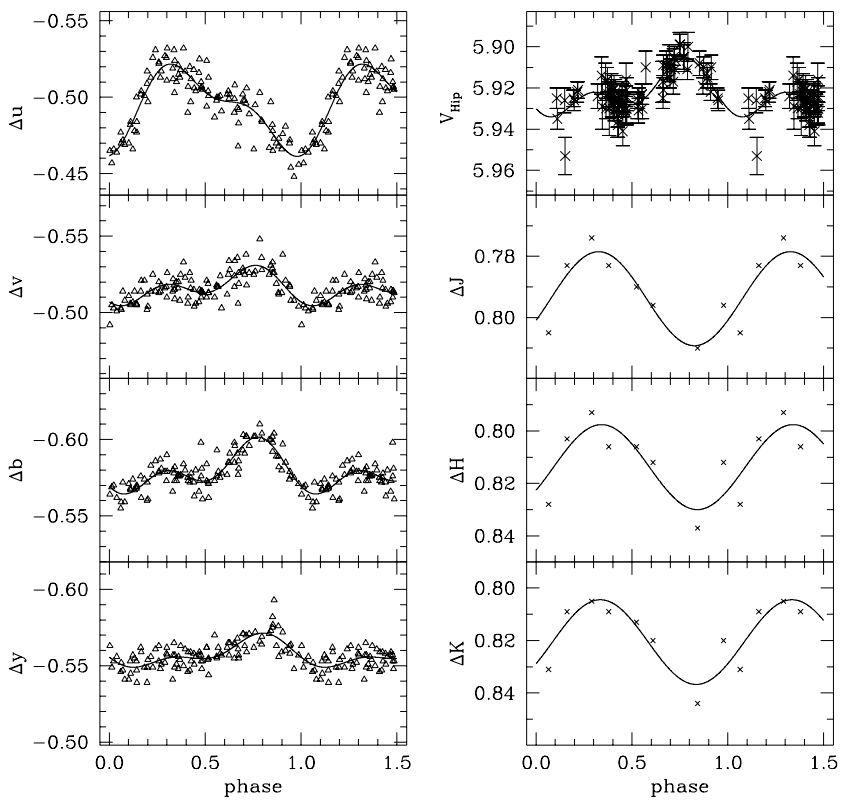

Fig. 3. Strömgren differential photometry by Adelman (1997) and Hipparcos light curve of 25 Sex. Near infrared light curves are from Catalano et al. (1998). Solid lines represent a leastsquare fitting of data with Eq. (1), as to the infrared ones we have neglected the $A_{2}$ term

\subsection{Sex $=H D 90044=H R 4082=H I P 50885$}

25 Sex is classified as a B9SiCrSr star. Its photometric variability ephemeris:

$\mathrm{JD}\left(u_{\min }\right)=2445659.000+4.37900 E$

were established by Manfroid \& Renson (1994). The validity of this period is confirmed by Adelman (1997) and also by Hipparcos photometry: a fitting of the Hipparcos and Adelman's b-filter variations with Eq. (1) shows that the primary maxima are the phase 0.776 and 0.774 respectively (Fig. 3).

The effective magnetic field of this star was measured by Bohlender et al. (1993), who could not establish the magnetic variability because of an unlucky phase coverage. Combining our with Bohlender et al. observations, we find that the effective magnetic field of 25 Sex changes sinusoidally between, roughly, 1 and $-1 \mathrm{kG}$ (Fig. 4).

The visible light variability of this star is rather complex. According to Adelman's (1997) observations (Fig. 3): $u$ variation shows the absolute maximum around the 0.3 phase and a secondary maximum around the 0.8 phase; $b$ shows the primary maximum at the 0.8 phase and the secondary maximum at 0.3 phase; $v$ and $y$ light curves resemble the $b$ variation even if with a very small $(\sim 0.01 \mathrm{mag})$ amplitude. Thus, light maxima show the phase of the $H_{\text {eff }}$ extrema and light minima have the null field phases. Much simpler are the near infrared light curves determined by Catalano et al. (1998) whose sinusoidal variation is almost in phase with $H_{\text {eff }}$ (Fig. 3).

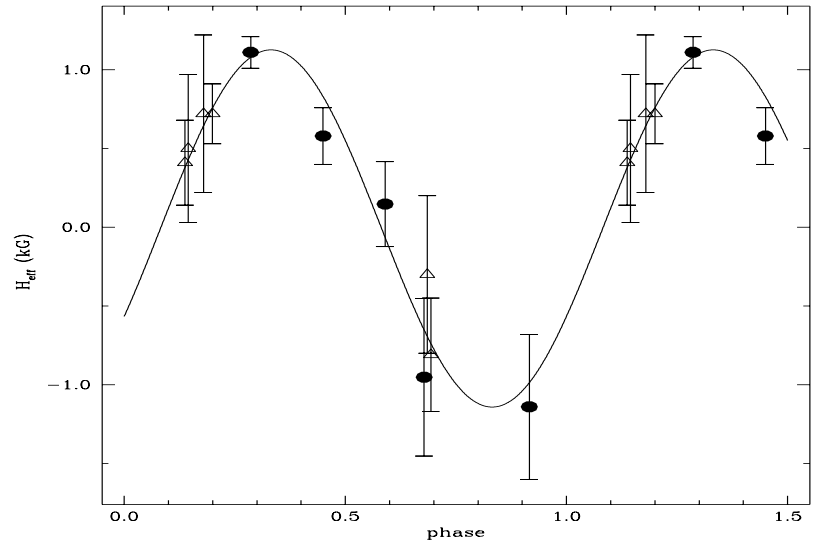

Fig. 4. Effective magnetic field of 25 Sex as a function of the rotational phase. Triangles represent the photopolarimetric measurements by Bohlender et al. (1993) and dots our spectropolarimetric observations. The solid line is sine function fit

\section{3. $E P$ UMa $=H D 96707=H R 4330=H I P 54540$}

In the General Catalogue of Ap and Am stars by Renson et al. (1991), EP UMa is classified as an A8Sr star with the "?" flag meaning that its peculiar nature has to be confirmed. Probably these authors did not consider conclusive the measurements by van den Heuvel (1971) of $H_{\text {eff }}$ ranging from -2.7 to $0.8 \mathrm{kG}$ with errors larger than $1 \mathrm{kG}$.

Johnson photometric observations of EP UMa were obtained by Winzer (1974), who suggested a variability period of 0.8183 days. Adelman et al. (1999) have obtained Strömgren photometric observations of EP UMa and concluded that this star presents single-wave light variations with a 3.5160 day period. The amplitude is $0.02 \mathrm{mag}$ in $b$ and $0.025 \mathrm{mag}$ in $y$ filter, with those in $u$ and $v$ probably smaller.

We have obtained two positive and four negative measurements of $H_{\text {eff }}$ confirming that EP UMa is a CP star. However, our measurements are not variable with the period determined by Adelman et al. Since double-wave light variations are commonly presented by $\mathrm{CP}$ stars whose magnetic field changes its sign, it is possible that EP UMa is also characterised by light curves with a double-wave. Adopting the period $P=2 \times 3.5160=7.0320$ days, our measurements of $H_{\text {eff }}$ show a sinusoidal variation. However, with this period there is a small shift between the Adelman et al. (1999) and Hipparcos light curves. Thus we have adopted the ephemeris:

$\operatorname{JD}\left(y_{\max }\right)=2441447.71+7.0286 E$

that let the Adelman and Hipparcos light curves to vary in phase (Fig. 5).

We find that our magnetic field measurements can be well fitted with a simple sine function (Fig. 6). With this period, light curves present two maxima at the phase of $H_{\text {eff }}$ extrema (Fig. 5).

It is worthy to note that the variability period of HD 200311 was determined in the same way by Wade et al. (1997). 


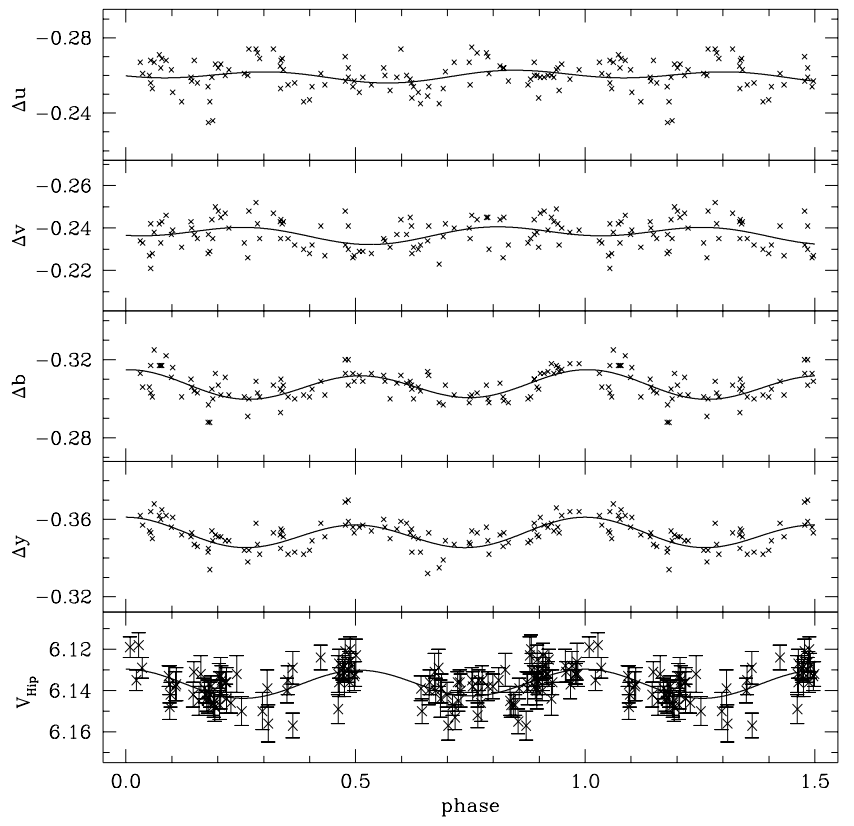

Fig. 5. Strömgren differential photometry by Adelman et al. (1999) and Hipparcos light curve of EP UMa

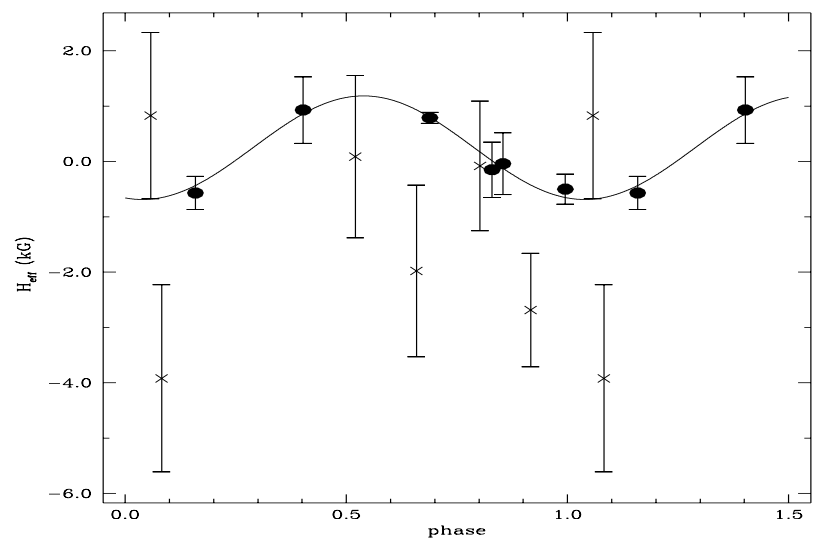

Fig. 6. Effective magnetic field of EP UMa as a function of the rotational phase. Our measurements (circles) have been fitted with a sine function. Van de Heuvel observations (crosses) are also reported

With the here suggested period, van den Heuvel (1971) measurements of the effective magnetic field are not in agreement with our measurements (Fig. 6). Further measurements of the effective magnetic field are then necessary to better define the behaviour of EP UMa.

\subsection{Vir $=H D 118022=H R 5105=H I P 66200$}

$78 \mathrm{Vir}$ is classified as an $\mathrm{A} 1 \mathrm{EuCr}$ star. Its photometric variability period has been determined by Catalano \& Leone (1994) as equal to 3.722084 days.

Figures 7 and 8 show the magnetic and photometric variability according to the ephemeris:

$\operatorname{JD}\left(H_{\mathrm{eff}}(\max )\right)=2434816.90+3.722084 E$.

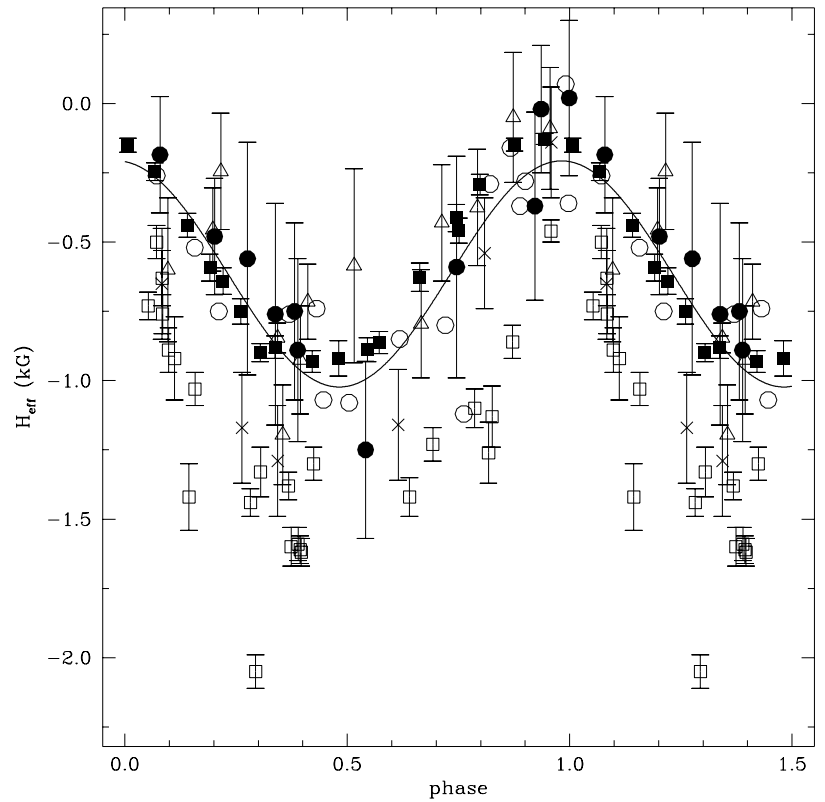

Fig. 7. Effective magnetic field of $78 \mathrm{Vir}$ as a function of the rotational phase. We report measurements by Preston (1969) (squares), Wolff \& Bonsack (1972) (crosses), Wolff (1978) (empty circles), Borra \& Landstreet (1980), Borra et al. (1981) (triangles), Wade et al. (2000) (filled squares) and by us (dots). Solid line represent a sinusoidal fit of these data with the exclusion of Preston and Wolff \& Bonsack observations
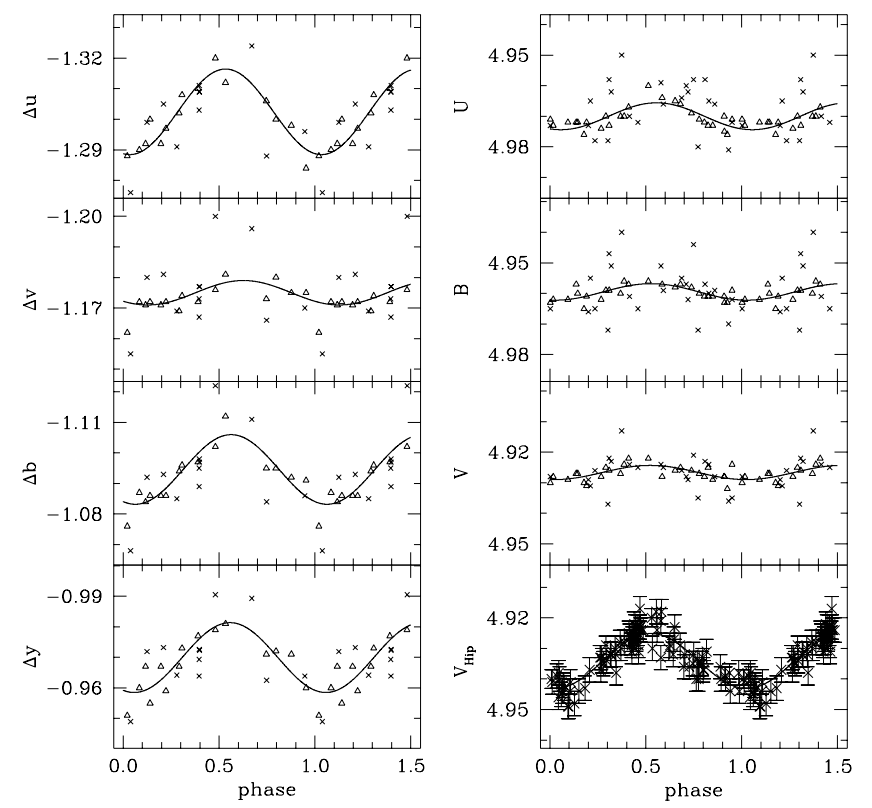

Fig. 8. Light variations of 78 Vir. Strömgren photometry is from Wolff \& Wolff (1971) (triangles) and Catalano \& Leone (1994) (crosses). Johnson photometry is from Stepień (1968) (crosses) and van Genderen (1971) (triangles). Hipparcos photometry shows clearly that light variations are not purely sinusoidal

We note that, within errors, our measurements of $H_{\text {eff }}$ are in agreement with the measurements by Wolff (1978), Borra \& Landstreet (1980), Borra et al. (1984) and Wade et al. (2000). In contrast, Preston (1969) and 
Wolff \& Bonsack (1972) spectropolarimetric measurements are systematically more negative (Fig. 7).

Light curves in Strömgren and Johnson photometric systems from several authors are almost sinusoidal functions and they show that 78 Vir is faintest when the effective magnetic field is null. Hipparcos photometry shows better than the other data that the light variation is not purely sinusoidal (Fig. 8).

Wade et al. (2000) noted that Catalano \& Leone photometric observations are not variable with the 3.7218 day period, that is necessary to get a magnetic field model consistent with the photopolarimetric measurements by Leroy et al. (1996). We note that adopting the 3.7218 day period, there is a 0.12 phase shift between Hipparcos light curves and the other light curves. Moreover, we find that adopting the 3.722084 day period, all the available measurements of $H_{\text {eff }}$ are in phase even if they present a different average value ${ }^{1}$. On the contrary, the 3.7218 day period is not representative of the variability for the oldest $H_{\text {eff }}$ measurements.

\section{5. $C S$ Vir $=H D 125248=H R 5355=H I P 69929$}

CS Vir is classified as an A9SrEuCr star. The spectroscopic variability of this star was established by Deutsch (1947) as equal to 9.295 days.

With the aim to determine a period which is accurate enough to relate the light and magnetic variability we have considered the photometric observations by Stibbs (1950), Maitzen \& Rakosch (1970), Maitzen \& Moffat (1972) and Pyper \& Adelman (1985). Moreover we have analysed the Hipparcos photometric data and our observations in the Strömgren system obtained in March 1991 with the $50 \mathrm{~cm}$ Danish telescope operating at ESO-LaSilla. Acquisition and reduction method of these photometric data is described in Catalano \& Leone (1993). Because of the different photometric systems, we have preferred to look for the period which gives an equal phase for the minimum of Stibbs', $B$ and $v$ light curves. We obtained the ephemeris:

$\mathrm{JD}\left(v_{\max }\right)=2433103.95+9.29545 E$.

Figure 9 shows the available photometric variations, with the differential photometric data over-plotted to the absolute photometry. $A_{0}$ coefficients of Eq. (1) were determined separately fitting any set of data.

Adopting the period determined here, we have phased our measurements of the effective magnetic field together with the measurements by Babcock (1951, 1958), Hockey (1969), Landstreet et al. (1975), Borra \& Landstreet (1980), Mathys (1991) and Mathys \& Hubrig (1997) (Fig. 10).

We note that the $U$ and $B$ light curves are single-waves which are out of phase with respect to the $H_{\text {eff }}$ variation. The $V$ variation is a double-wave whose two maxima show the phase of $H_{\text {eff }}$ extrema, the maximum coinciding with

\footnotetext{
${ }^{1}$ Referring to $\beta \mathrm{CrB}$, we will discuss on systematic differences between different data sets of $H_{\text {eff }}$.
}
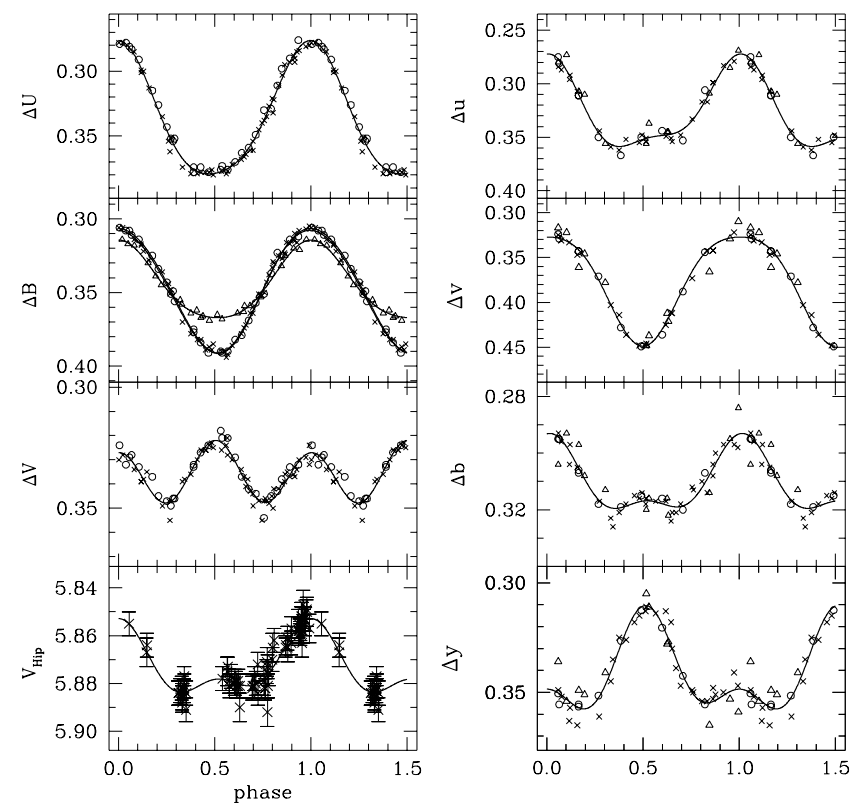

Fig. 9. Light variation of CS Vir. Strömgren photometric data are from Maitzen \& Moffat (1972) (crosses), Pyper \& Adelman (1985) (triangles) and Catalano et al. (1992) (circles). Empty circles represent our photometric data. Among Johnson photometric data, empty circles represent Maitzen \& Rakosch (1970), crosses represent Maitzen \& Moffat (1972). Triangles represent Stibbs (1950) data. Hipparcos light curves is also reported

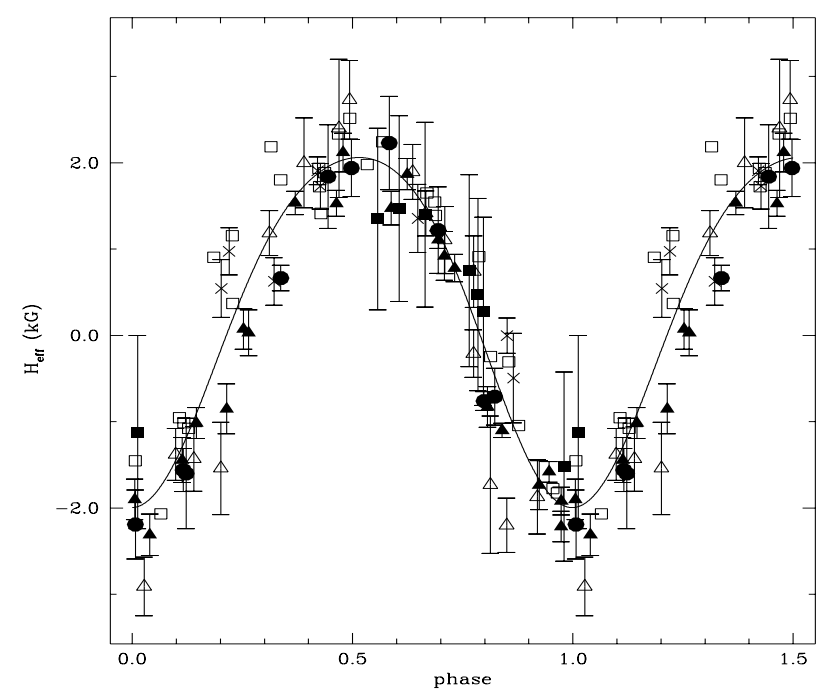

Fig. 10. Variability of the effective magnetic field of CS Vir with the rotational phase. Measurements are by Babcock (1951) (empty squares), Babcock (1958) (filled squares), Hockey (1969) (crosses), Landstreet et al. (1975) and Borra \& Landstreet (1980) (empty triangles), Mathys (1991), Mathys \& Hubrig (1997) (filled triangles) and by us (filled circles)

the positive magnetic extremum is slightly larger than the other one. It is worthy to note that the amplitudes of the $V$ and $y$ variations are comparable, and that the $y$ light curve shows a primary maximum at the phase of the negative magnetic extremum and that the secondary maximum 

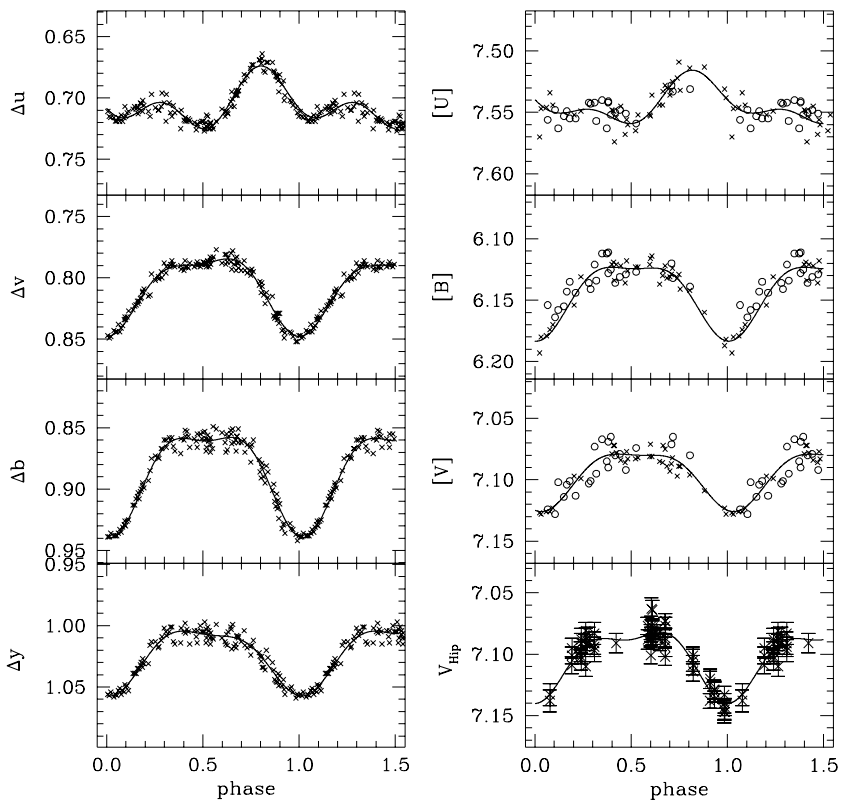

Fig. 11. North \& Adelman (1995) light curves of FF Vir in the Strömgren and Geneva photometric systems. Circles represent Catalano \& Leone (1990) differential photometry, in the Johnson system, appropriately shifted. Hipparcos light curve is also reported

is much smaller than the primary one. Moreover, the $u$ variation presents a secondary maximum that is not observed in the $U$ variation.

According to Catalano et al. (1992), the near infrared variability resemble the $V$ light curve.

\section{6. $F F$ Vir $=H D 126515=H I P 70553$}

The A9CrSr star FF Vir was found to be a magnetic star by Babcock (1958).

The variability period of the magnetic field modulus was determined by Preston (1970) as equal to 130 days. North \& Adelman (1995) from photometric data in the Strömgren and Geneva systems concluded that the variability period is $129.99 \pm 0.04$ days. Combining their own and Preston measurements of the magnetic modulus, Mathys et al. (1997) found a period equal to $129.95 \pm$ 0.02 days.

Starting from this value of the period, we note that the minima of North \& Adelman and Hipparcos light curves show the same phase for the period: $P=129.9474$ days. Thus, we have adopted the ephemeris:

$\operatorname{JD}\left(v_{\min }\right)=2436959.0+129.9474 E$

to phase the magnetic and light variations.

Figure 11 shows Strömgren and Geneva photometry obtained by North \& Adelman (1995) together with Catalano \& Leone (1990) UBV photometry and Hipparcos data.

Figure 12 shows our measurements of $H_{\text {eff }}$ together with the values by Babcock (1958), Preston (1970),

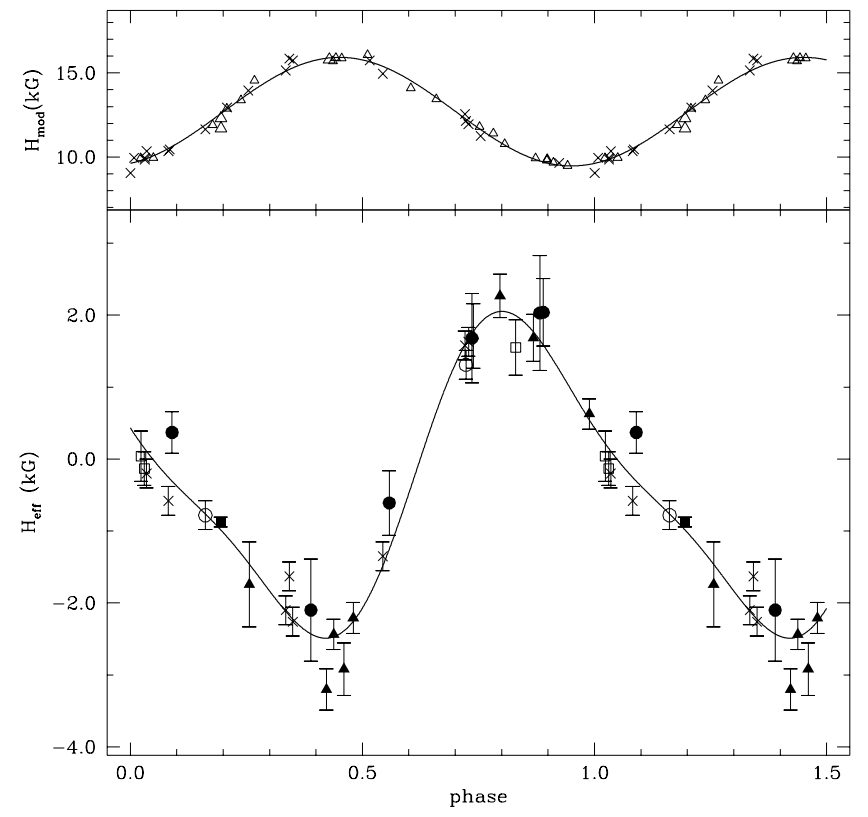

Fig. 12. Measurements of $H_{\text {eff }}$ for FF Vir by Babcock (1958) (open circles), Preston (1970) (crosses), van de Heuvel (1971) (empty square), Mathys (1994), Mathys \& Hubrig (1997) (filled triangles), Wade et al. (2000) (filled squares) and by us (filled circles). Solid line represents a fit of data with Eq. (1). Measures of the magnetic field modulus are by Preston (1970) (triangles), Mathys \& Lanz (1992) and Mathys et al. (1997) (circles). The solid line is a sinusoidal fit of data

van de Heuvel (1971), Mathys (1994), Mathys \& Hubrig (1997) and Wade et al. (2000). In the same figure $H_{\text {mod }}$ measurements of FF Vir by Mathys et al. (1997) are plotted. It appears that $H_{\text {eff }}$ is null when $H_{\text {mod }}$ is minimum and that $H_{\text {mod }}$ maximum shows the phase of the $H_{\text {eff }}$ minimum.

We note that the $u$ variation shows the primary maximum at phase 0.8 , where $H_{\text {eff }}$ is maximum, and the secondary maximum at phase 0.3 without any relation with the $H_{\text {eff }}$ or $H_{\text {mod }}$ variation. Longward of the $u$ filter, light curves resembles the variation of the magnetic field modulus.

\section{7. $\beta \operatorname{CrB}=H D 137909=H R 5447=H I P 75695$}

$\beta \mathrm{CrB}$ is an $\mathrm{A} 8 \mathrm{SrEu}$ star whose ephemeris:

$\mathrm{JD}($ magnetic $\max )=2434204.70+18.4868 E$

were established by Kurtz (1989) analysing all the $H_{\text {eff }}$ measurements published from Babcock (1958) to Borra et al. (1981). Within errors, this period is consistent with the light variability period determined by Adelman et al. (1992): $P=18.487$ days.

We have repeated Kurtz's exercise adding the observations by Mathys (1991) (11 measurements), Mathys \& Hubrig (1997) (4 measurements), Hildebrandt et al. (1997) (2 measurements), Wade et al. (2000) (17 measurements) and our (13 measurements) spanning 


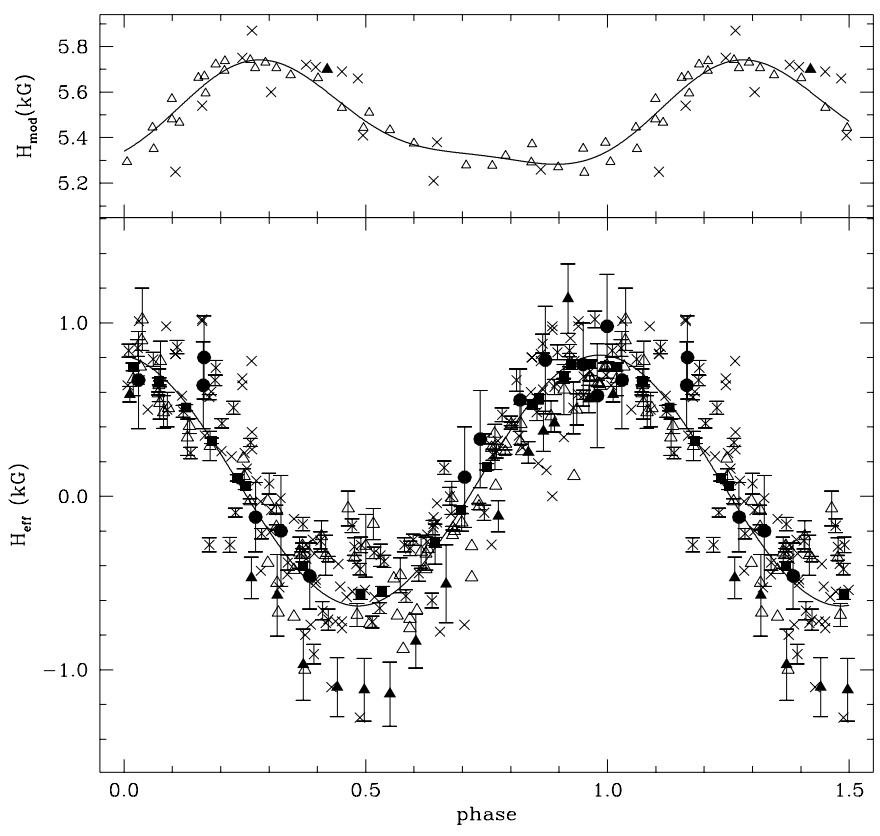

Fig. 13. Measures of the effective magnetic field of $\beta \mathrm{CrB}$. Spectropolarimetric measures with photographic plates are by Wolff \& Wolff (1970), Wolff \& Bonsack (1972) and Wolff (1978) (crosses), Slovak (1982) (open squares), Babcock (1958) (open circles), Vogt et al. (1980) (stars) and Hildebrandt et al. (1997) (filled squares). Open triangles represent the photopolarimetric measurements by Landstreet et al. (1975), Borra \& Vaughan (1977), Brown \& Landstreet (1981) and Borra et al. (1984). CCD spectropolarimetric measurements are by Mathys (1991) (filled triangles) and by us (filled circles). Solid line represents a least-squares fit with a sine function. Magnetic field modulus has been measured by Wolff \& Wolff (1970) (crosses), Mathys \& Lanz (1992) (filled triangles) and Mathys et al. (1997) (open triangles). Solid line represent a fit of Mathys et al. (1997) data with Eq. (1)

$\sim 17000$ days. A sine fit of all these data gives again the period determined by Kurtz, period error (defined as the variation that increases the $\chi^{2}$ of a unit) is 0.0002 days.

Figure 13 shows the $H_{\text {eff }}$, previously quoted, phased with Kurtz's ephemeris. Wade et al. (2000) noted that their measurements of $\beta \mathrm{CrB}$ are closer to the photopolarimetric (by Borra, Landstreet et al.) than to the spectropolarimetric results by Mathys, with the exception that LSD data do not present the hump near the magnetic minimum which is present in the polarimetric measurements. In spite our method of measuring the effective magnetic field is practically equal to Mathys method (Leone et al. 2000), we have obtained different results, and our measurements are (within errors) not different than Wade et al. (2000) results.

Preston \& Sturch (1967) suggested the possibility that $H_{\text {eff }}$ of the binary star $\beta \mathrm{CrB}$ is not only variable with the rotational period but also with the orbital one. Thus, we have phased all the previous magnetic observations with the orbital period (= 3858.13 days) determined by North et al. (1998). Starting from the null value of the orbital phase, all the $H_{\text {eff }}$ measurements within 0.1 phase
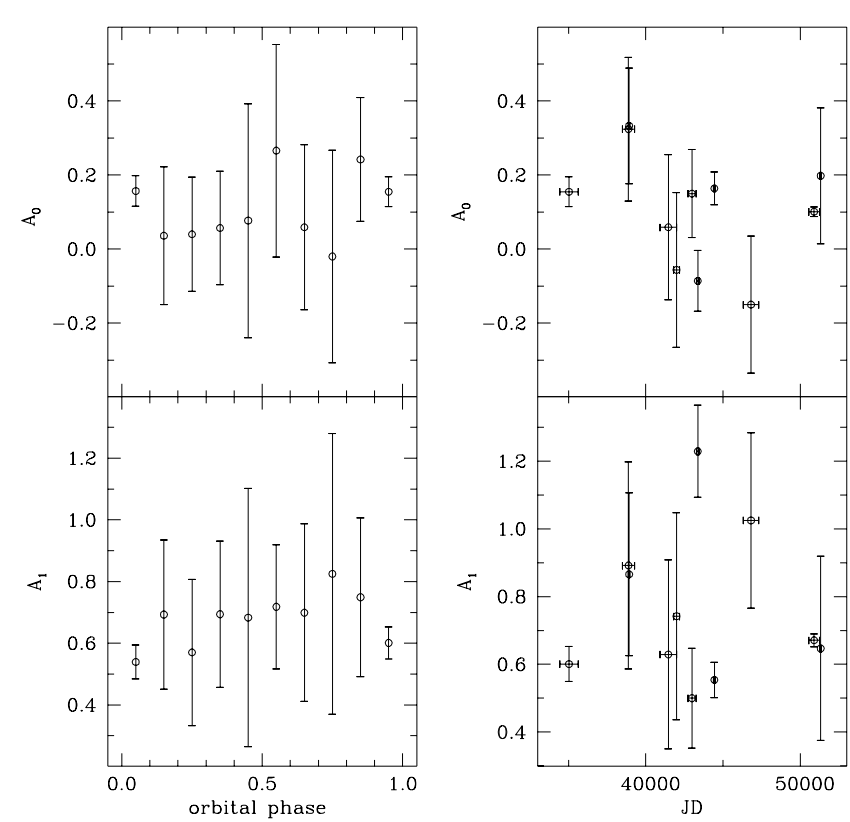

Fig. 14. Phasing the $H_{\text {eff }}$ measurements of $\beta \mathrm{CrB}$ with the orbital period, data within 0.1 phase intervals have been phased with the orbital period and than fitted with a sine function. Left panel shows the amplitude $A_{1}$ and average value $A_{0}$ as a function of the orbital phase. Right panel shows the amplitude and the average value for each single data set

intervals have been phased assuming the 18.4868 day rotational period. Each sub-sets of data have than been fitted, following Kurtz, with a sine function $\left(A_{2}=0\right)$. The left panel of Fig. 14 shows the amplitude $\left(A_{1}\right)$ and the average value $\left(A_{0}\right)$ with the associated errors for each sub-set of data. We note that errors are very large and that there is no evidence of variability with the orbital period. Thus, we have performed a sinusoidal fit of the data sets given by the different authors. The right panel shows the large differences between the parameters, we conclude that no secular variability is clearly evident and that differences are due to the different observational methods.

Systematic differences between the considered data sets could be at the origin of the large error associated with the determined variability period of $\beta \mathrm{CrB}$. Similarly, systematic differences are also probably at the origin of the large scatter observed in the measurements of the effective magnetic field of 78 Vir. For this star, Wolff et al. measurements present a clear $-0.5 \mathrm{kG}$ shift with respect to the other data sets (Fig. 7).

Differently than for FF Vir, $\beta$ CrB presents the primary maximum of $H_{\text {mod }}$ at the same phase than $H_{\text {eff }}$ null (Fig. 13). The $u$ light curve shows a double-wave light variation with minima during $H_{\text {eff }}$ extrema. The $V_{\text {Hip }}$ photometric variation is in phase with the vby light curves (Fig. 15) and resemble the $H_{\text {eff }}$ variation.

\section{Conclusions}

We have obtained spectropolarimetric measurements of the effective magnetic fields for a sample of chemically 


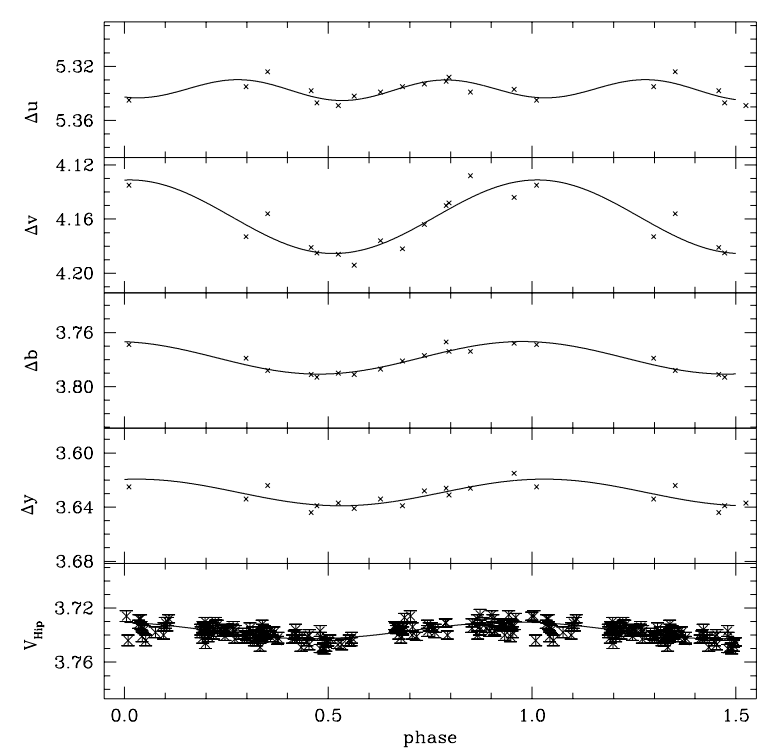

Fig. 15. Strömgren photometry by Pyper \& Adelman (1985) and Hipparcos light curve of $\beta \mathrm{CrB}$. Because of the very small amplitude, only $V_{\text {Hip }}$ values with error smaller than 0.005 mag have been considered to plot the Hipparcos light curve. Solid lines represent a sinusoidal fit of data

peculiar stars. The magnetic variability of 25 Sex and EP UMa have been ascertained for the first time. Starting from values given in the literature, the accuracy of variability periods for CS Vir and FF Vir have been improved.

Our measurements of the effective magnetic field combined with data from the literature have been used to establish the phase relations of the magnetic and light variabilities.

In principle, light variations are expected to be, indirectly, due to the magnetic field. Element diffusion (Michaud 1970) is, up-to-now, the most reliable explanation for anomalous abundances, and being ions diffusion strongly dependent on the magnetic field strength and geometry, it results in a non homogeneous distribution of elements on the stellar surface. Leckrone (1974) suggested that in metal rich photospheric regions the ultraviolet flux is blocked and than redistributed to the longest wavelengths so that light variations are due to the stellar rotation. In this hypothesis, out of phase variations are expected between ultraviolet and visible light curves and an wavelength interval with constant flux emission exists in between, the so called null wavelength.

However, Leckrone's suggestion is not straightly applicable to the here considered CP stars, with the exception of EP UMa. We find that:

- 53 Cam presents a very complex light variability, with the phase of light minima changing with the wavelength;

- 25 Sex shows double-wave light curves in the visible and single-wave light curves in the near infrared;
- CS Vir light curves are single-wave shortward and double-wave longward of $\sim 4500 \AA$. Moreover, the $V$ light curve is a double-wave and the $y$ is practically single-wave, and the $u$ light curve presents a secondary maximum which is absent in the $U$ curve;

- FF Vir $u$ variation is not specular with respect to the longest wavelength variations, and

- $\beta$ CrB presents a double-wave variation in the $u$ filter and a single-wave variation in the other Strömgren filters.

We conclude that flux redistribution from the ultraviolet to the visible wavelengths, in metal rich regions, cannot alone explain the observed light variability and that further mechanisms have to be invoked. In the literature we find that:

- Bonsack (1979) measured a local line-blocking that can justify half of the HD 24712 light variability. This mechanism is certainly important to explain the different behaviour of the CS Vir light curves within the broad $V$ and narrow $y$ pass-band filters;

- Trasco (1972) suggested that the surface of a magnetised star is not iso-thermal and that a light modulation is expected with the rotation. This mechanism could be important for 25 Sex presenting a very complex light behaviour in the visible and a much simpler variation in the near infrared. Line-blocking is certainly not important in the infrared spectrum of a B9 star, however the magnetic field could affect the structure of the outer layers.

Acknowledgements. This research has been supported by the Italian Ministero dell'Università e della Ricerca Scientifica e Tecnologica and by the Regione Sicilia which are gratefully acknowledged. This research has made use of the SIMBAD database, operated at CDS, Strasbourg, France. Thanks are due to Mr. Giovanni Gentile for the realisation of the polarimetric module.

\section{References}

Adelman, S. J. 1997, A\&AS, 122, 249

Adelman, S. J., Dukes, R. J., \& Pyper, D. M. 1992, AJ, 104, 314

Adelman, S. J., Rayle, K. E., \& Pi, C.-L. M. 1999, A\&AS, 136, 379

Babcock, H. W. 1947, ApJ, 105, 105

Babcock, H. W. 1949, Observatory, 69, 191

Babcock, H. W. 1951, ApJ, 114, 1

Babcock, H. W. 1958, ApJS, 3, 141

Babcock, H. W. 1960, ApJ, 132, 521

Bagnulo, S., Landolfi, M., \& Landi Degl'Innoccenti, M. 1999, A\&A, 343, 865

Bohlender, D., Landstreet, J. D., \& Thompson, I. B. 1993, A\&A, 269, 355

Bonsack, W. 1979, PASP, 91, 648

Borra, E. F., Edwards, G., \& Mayor, M. 1984, ApJ, 284, 211

Borra, E. F., Fletcher, J. M., \& Poeckert, R. 1981, ApJ, 247, 569 
Borra, E. F., \& Landstreet, J. D. 1973, ApJ, 185, 139

Borra, E. F., \& Landstreet, J. D. 1977, ApJ, 212, 141

Borra, E. F., \& Landstreet, J. D. 1980, ApJS, 42, 421

Borra, E. F., \& Vaughan, A. H. 1976, ApJ, 210, 145

Borra, E. F., \& Vaughan, A. H. 1977, ApJ, 216, 462

Brown, D. N., \& Landstreet, J. D. 1981, ApJ, 246, 899

Catalano, F. A., \& Leone, F. 1990, A\&AS, 89, 121

Catalano, F. A., \& Leone, F. 1993, A\&AS, 97, 501

Catalano, F. A., \& Leone, F. 1994, A\&AS, 108, 595

Catalano, F. A., Kroll, R., \& Leone, F. 1992, A\&A, 263, 203

Catalano, F. A., Leone, F., \& Kroll, R. 1998, A\&AS, 131, 63

Deutsch, A. J. 1947, ApJ, 105, 283

Deutsch, A. J. 1957, AJ, 62, 137

Elkin, V. G. 1996, A\&A, 312, 5

European Space Agency, 1997, Hipparcos Catalogue, SP-1200

Hildebrandt, G., Scholz, G., Rendtel, J., Woche, M., \& Lehmann, H. 1997, AN, 318, 291

Hill, G. M., Bohlender, D. A., Landstreet, J. D., et al. 1998, MNRAS, 297, 236

Hockey, M. S. 1969, MNRAS, 142, 543

Jarzebowski, T. 1960a, AcA, 10, 42

Jarzebowski, T. 1960b, AcA, 10, 237

Kurtz, D. W. 1989, MNRAS, 238, 261

Landolfi, M., Landi Degl'Innocenti, E., Landi Degl'Innocenti, M., \& Leroy, J.L., 1993, A\&A, 272, 285

Landolfi, M., Bagnulo, S., \& Landi Degl'Innocenti, M. 1998, A\&A, 338, 111

Landstreet, J. D., Borra, E. F., Angel, J. R. P., \& Illing, R. M. E. 1975, ApJ, 201, 624

Leckrone, D. S. 1974, ApJ, 190, 319

Leone, F., Catanzaro, G., \& Catalano, S. 2000, A\&A, 355, 315

Leroy, J.-L., Landolfi, M., \& Landi Degl'Innocenti, E. 1996, A\&A, 311, 513

Maitzen, H. M., \& Rakosch, K. D. 1970, A\&A, 7, 10

Maitzen, H. M., \& Moffat, A. F. J. 1972, A\&A, 16, 385

Manfroid, J., \& Renson, P. 1994, A\&A, 281, 73

Mathys, G. 1991, A\&AS, 89, 121

Mathys, G. 1993, Magnetic field diagnosis through spectropolarimetry, in Peculiar versus normal phenomena in A-type and related stars, ed. M. M. Dworetsky, F. Castelli,
\& R. Faraggiana, IAU Colloq. No. 138, ASP Conf. Ser. 44, 232

Mathys, G. 1994, A\&AS, 108, 547

Mathys, G., \& Hubrig, S. 1997, A\&AS, 124, 475

Mathys, G., Hubrig, S., Landstreet, J. D., Lanz, T., \& Manfroid, J. 1997, A\&AS, 123, 353

Mathys, G., \& Lanz, T. 1992, A\&A, 256, 169

Michaud, G. 1970, ApJ, 160, 641

Musielok, B., Lange, D., Schöneich, W., et al. 1980, AN, 301, 71

North, P. 1998, A\&A, 334, 181

North, P., \& Adelman, S. J. 1995, A\&AS, 111, 41

North, P., Carquillat, J.-M., Ginestet, N., Carrier, F., \& Udry, S. 1998, A\&AS, 130, 223

Preston, G. W. 1969, ApJ, 158, 243

Preston, G. W. 1970, ApJ, 160, 1059

Preston, G. W., \& Stepień, K. 1968, ApJ, 151, 583

Preston, G. W., \& Sturch, C. 1967, The magnetic field of $\beta$ Coronae Borealis, in The magnetic and related stars, ed. R. C. Cameron, Mono Book Corporation, Baltimore, 111

Pyper, D. M., \& Adelman, S. J. 1985, A\&AS, 59, 369

Renson, P., Gerbaldi, M., \& Catalano, F. A. 1991, A\&AS, 89, 429

Slovak, M. H. 1982, ApJ, 262, 282

Stepień, K. 1968, ApJ, 154, 945

Stepień, K. 1978, AcA, 28, 555

Stibbs, D. W. N. 1950, MNRAS, 110, 395

Trasco, J. D. 1972, ApJ, 171, 569

van den Heuvel, E. P. J. 1971, A\&A, 11, 461

van Genderen, A. M. 1971, A\&A, 14, 48

Vogt, S. S., Tull, R. G., \& Kelton, P. W. 1980, ApJ, 236, 308

Wade, G. A., Elkin, V. G., Landstreet, J. D., \& Romanyuk, I. I. 1997, MNRAS, 292, 748

Wade, G. A., Donati, J.-F., Landstreet, J. D., \& Shorlin, S. L. S. 2000, MNRAS, 313, 851

Winzer, J. E. 1974, Thesis, Univ. of Toronto

Wolff, S. C. 1978, PASP, 90, 412

Wolff, S. C., \& Bonsack, W. K. 1972, ApJ, 176, 425

Wolff, S. C., \& Wolff, R. J. 1970, ApJ, 160, 1049

Wolff, S. C., \& Wolff, R. J. 1971, AJ, 76, 422 